\title{
The Children's Machines: Handheld and Wearable Computers Too
}

\author{
Bakhtiar Mikhak, Fred Martin, Mitchel Resnick, Robbie Berg, \& Brian Silverman \\ MIT Media Laboratory, E15-001, 20 Ames Street, Cambridge MA 02139 \\ (mikhak, fredm, mres, rberg, bss)@media.mit.edu
}

\begin{abstract}
In this paper we describe the material of a construction kit designed to allow children to build their own handheld and wearable devices to meet their interests and passions. Children don't work with these machines, they learn, play and grow with them. Informed by the types of projects that children have done with this material in the context of educational research in science and engineering, we present a few scenarios for why children would build their own handheld or wearable computational devices. We believe that these application scenarios and their appeal to children are strong evidence for why we should rethink the design of computational devices, particularly for children.
\end{abstract}

\section{Introduction: Handheld/Wearable Computers and Children}

"Across the world children have entered a passionate and enduring love affair with the computer. What they do with computers is as varied as their activities...The love affair involves more than the desire to do things with computers. It also has an element of possessiveness and, most importantly, of assertion of intellectual identity. Large numbers of children see the computers as "theirs"-as something that belongs to them, to their generation." 1

These images of the relationship between children and computers are what inspire the present work. In the past few years, small computational toys such as Gameboy ${ }^{\mathrm{TM}}$, Tamagochi $^{\mathrm{TM}}$, Friend.link ${ }^{\mathrm{TM}}$, and Groove Connection ${ }^{\mathrm{TM}}$ have taken our children's relationship with computers to a new level. They now play and act out nurturing games, keep in touch and interact with their friends, and more broadly learn about themselves and the world with and through their machines. The diversity in children's interests in and expectations of their handheld computers is what has produced a growing market for a large number of specialized devices.

However, another way to address this demand is to provide children with a set of tools to build and reconfigure their own handheld and wearable devices. We have taken this approach because it also provides a natural context for many new learning opportunities. In the process of building devices that they care about, children get a

${ }^{1}$ The Children's Machine by Seymour Papert (page ix) 
chance to look beyond and into the "black boxes" that surround them, and become proficient designers and critics of the tools they live and think with.

In this paper, we describe a Handheld/Wearable Computer Construction Kit, and propose a number of scenarios for what it would look like and what activities it could support. We will present a mixture of projects in various states of development. Some of the technologies we describe have been used extensively by children in a variety of classroom settings. Others are more forward looking and have only been used in our research lab. We discuss all of these to give a sense of activities for children in the coming years.

In other published work, we have focused more on the educational aspects of this work than we do so here. Earlier versions of this work, known as programmable bricks, was used primarily in robotic design with grade school children (Martin, 1988) and with university students (Martin, 1994). The latest work is part of our Beyond Black Boxes initiative, which encourages children to design scientific instruments for their own investigations (Resnick, et. al., in press; Martin, et. al. in press). Here, we will emphasize a collection of technologies most relevant to the ubiquitous computing community. Over the next year, we plan to have an extensive analysis of children's experiences in the activities we propose here.

\section{The Handheld/Wearable Computer Construction Kit}

\subsection{Programmable Bricks}

In earlier work we developed the Programmable Brick, a hand-held computer brick that could control motors, receive information from sensors, and run a program written in a dialect of the Logo programming language. Our first Programmable Brick was used with fifth-grade children who constructed games and play environments for small mobile robots built from it. This work focused on children's relationships with the cybernetic playthings that they had constructed (Martin, 1988).

In later work, we formed a core set of activities for exploring engineering design (Martin, 1996) and mathematics at the elementary school level (Hayward, 1995). This work served as a foundation for the LEGO Group's recent launch of the LEGO Mindstorms product, a robot design kit marketed both at the retail level and for educational use. 


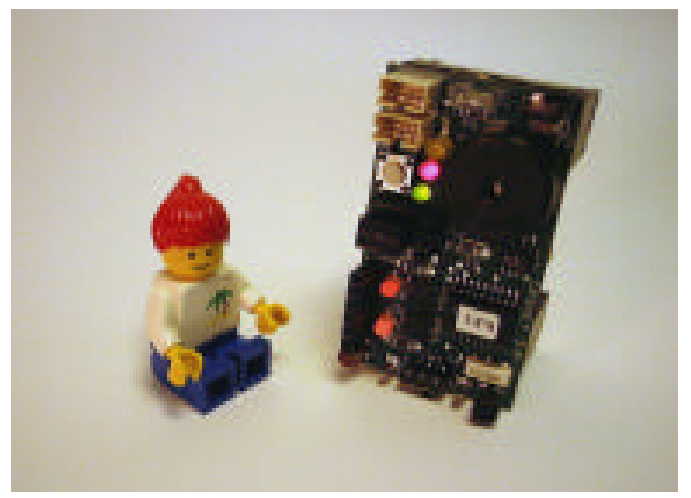

Fig. 1. A LEGO "Mini Fig" and the MIT Cricket (right)

Recently, we have designed the Cricket (Fig. 1), a tiny version of the Programmable Brick concept. The Cricket has fewer features than our earlier Bricks - for instance, it can directly power only two motors and receive data from two sensors-but because Crickets are so small, they open up new possibilities. Also, Crickets are designed with built-in infrared communications capability, and our latest versions include a multi-purpose "Bus Port," to which literally dozens of Cricket peripherals may be simultaneously connected (Martin et. al., 1999, in press).

\subsection{Cricket Peripherals}

As part of the Beyond Black Boxes work, we are developing a collection of sensors and output devices that can be used as building blocks by children in their projects. The Cricket Bus Port has opened up a whole new range of possibilities, starting with the fact that it once and for all ends the argument about how many sensor and actuator ports a programmable brick should have. Previous versions of our bricks had typically four motor ports and variously four, six, or more sensor inputs. With the Bus Port, the Cricket itself can have just two sensor inputs and two motor drivers, and additional devices may be connected to the Bus Port.

Much recent work has been devoted to the conception and development of new Bus Devices. Our present list of such Cricket peripherals includes:

- Heart Rate Monitor-Based on the Polar® exercise sensor, this device automatically tabulates a pulse measurement, returning a beats-per-minute reading that is updated every six seconds.

- 3-Digit Numeric Display-A large-character LED display, capable of displaying numeric values sent from the Cricket.

- Galvanic Skin Response (GSR) Sensor-A device that measures the resistance of skin, and provides a simple measure of excitement or stress.

- IR Proximity Sensor-Based on sensors used in public restrooms to detect (e.g.) your hands underneath the faucet, this device provides an accurate measurement to the nearest object within a range of $15 \mathrm{~cm}$ to nearly a meter. 
- Voice Recorder-Based on technology used in answering machines and personal audio memo recorders, this device lets children record a number of sound snippets which may be individually played back under program control.

- MIDI Boat-A high quality, compact MIDI synthesizer that will generate music from instructions supplied by the Cricket.

- Motorola ${ }^{\circledR}$ CreataLink ${ }^{\mathrm{TM}}$ _An interface to a national Motorola 2-way paging system, this technology allows Crickets to transmit and receive short data messages when located outdoors or in other remote locations.

\subsection{Software Environments}

An important dimension of the Handheld/Wearable Computer Construction Kit is the environment that children use to design the behaviors of their projects. We have two primary software environments: a text-based Logo language implementation, and an iconic version of Logo.

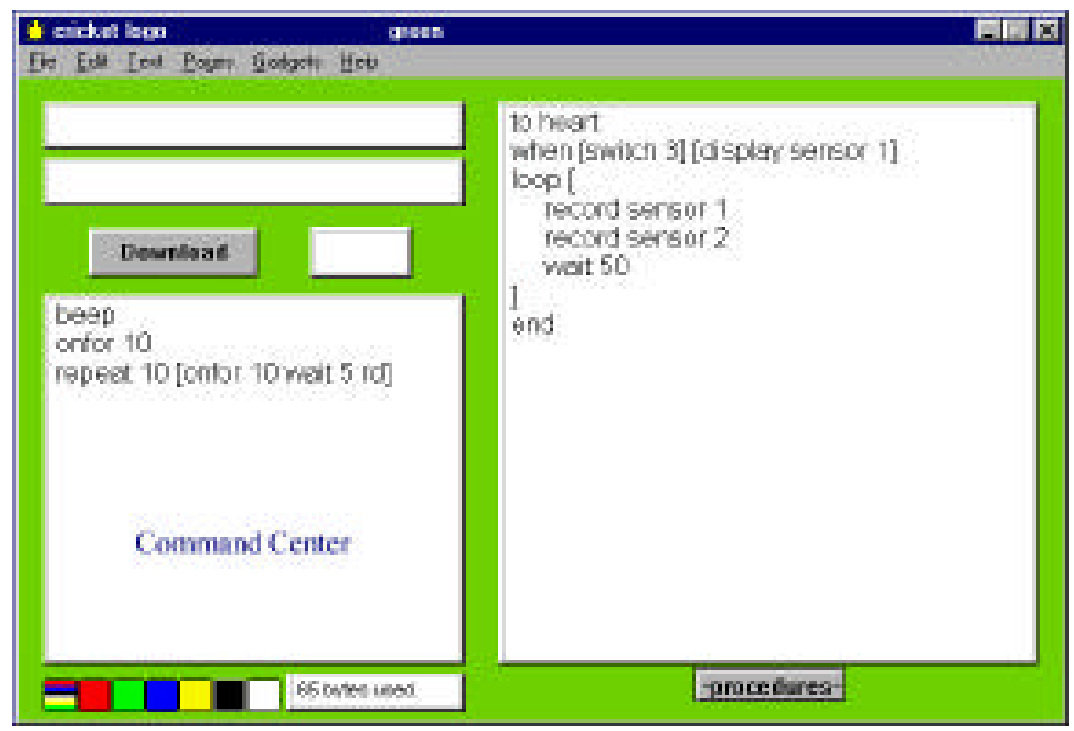

Fig. 2. Cricket Logo software interface. In the lower right is the command center; the Cricket immediately executes instructions typed in this window. On the right half of the screen is the procedures window; when the download button is clicked, all programs in the procedures window are transmitted to the Cricket.

Like traditional Logo software with screen turtles, our Cricket Logo (Fig. 2) includes a command center, an interaction window that acts as the main interface to the computational system. In a screen Logo, a child might type "fd 20 " to make the turtle move forward 20 turtle-steps; in Cricket Logo, a child would type "onfor 10 " to turn on a motor for one second. The facile, immediate feedback of the 
command center is critical to providing a powerful yet playful environment for learning how things work.

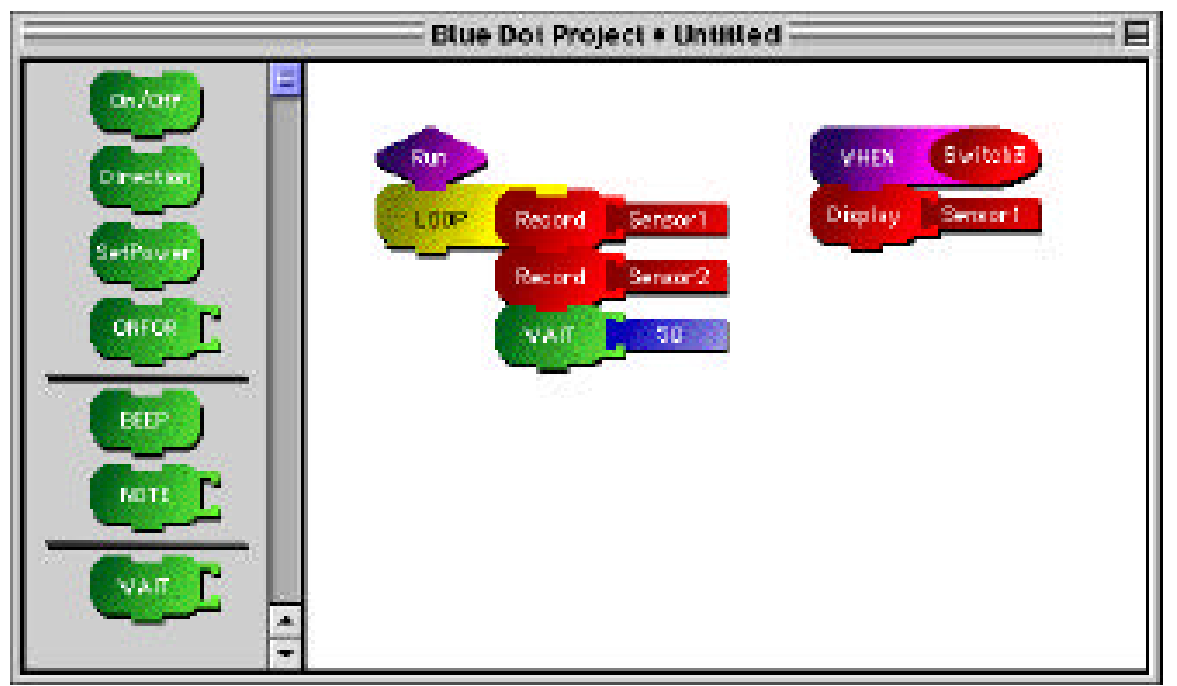

Fig. 3. Logo Blocks software interface. Program blocks are dragged off the palette on the left column and assembled into a program in the main screen window.

Our iconic environment, dubbed "Logo Blocks," is shown in Fig. 3. With Logo Blocks, children have visual color and shape cues that help them construct syntactically correct programs. All of the Logo commands are inside of blocks that may be dragged off of a palette and dropped into their program. We have found that many children who were frustrated or uninterested in traditional Logo programming have become quite engaged in programming using Logo Blocks.

Our plans are to bring these two distinct software systems into one. We are presently working on a version of Logo Blocks that will include "open code" blocks, into which one can type a traditional Logo program. Then after closing up the block, it will appear in the Logo Blocks palettes, ready to use as if it were any other "primitive" block.

\section{Application Scenarios}

This section presents applications inspired by projects that children have done with Crickets and Cricket peripherals in workshops and classrooms as part of the Beyond Black Boxes project. The top level goal of this project is to rethink science and engineering education fundamentally by developing a comprehensive set of tools, hardware and software, and sample projects that would allow children to build their own scientific instruments for investigation they care about. For further discussion of the learning value of these activities, please see Resnick et. al., 1999 (in press). 


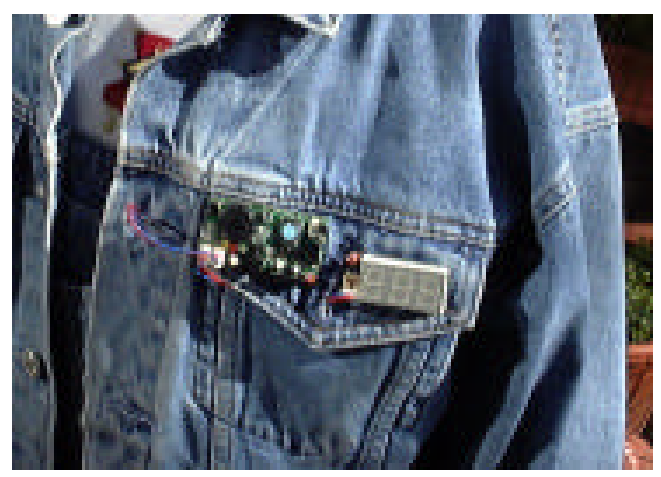

Fig. 4. Staying Cool

\subsection{Staying Cool}

Consider the wearable computer shown in Figure 4. It contains a heart rate sensor, a body temperature sensor, a 3-digit numeric display, and a touch switch. The computer records both sensors' values during the course of the day at a specified frequency. The program for the project, written in Logo Blocks, is shown earlier in Fig. 3. The set-up performs the following actions:

- The main program, on the left side, is triggered when the Cricket's RUN button is pressed.

- With the yellow LOOP block, three subsequent actions are looped indefinitely:

- Sensor 1, the instantaneous heart-rate reading, is captured and stored in the Cricket's data memory.

- Sensor 2, the temperature, is captured and stored.

- A delay of 5 seconds is performed before the loop recycles.

The 3-digit numeric display is controlled by the piece of the program on the right side of the screen. The WHEN block sets up a background monitoring process that executes in parallel to the main data-collection procedure. Whenever Switch 3 (a button carried in the pocket) is pressed, the current value of Sensor 1 (the heart-rate monitor) is presented on the numeric display.

It is instructive to compare this program with that written in Cricket Logo (Fig. 2). We have found that younger children compose such a program on their own more often and more easily in Logo Blocks. However, as they gain more experience and write longer programs they would benefit from a programming environment that takes advantage of the strengths of text and graphics both. 


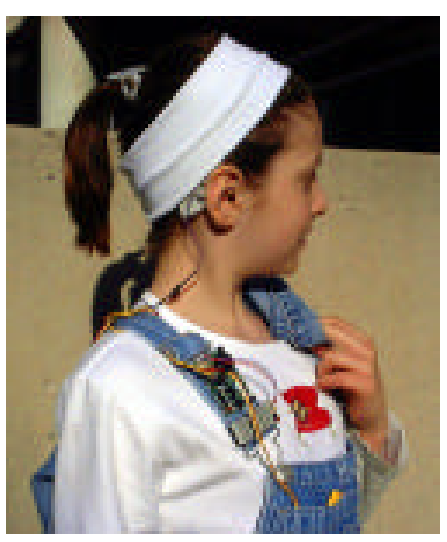

Fig. 5. Digital Mood Ring

\subsection{Digital Mood Rings}

The Digital Mood Ring consists of a Cricket, a galvanic skin response (GSR) sensor, and a display (Fig. 5). The sensor can be incorporated into one's clothing and the display can be playfully presented such that people can tell how stressed or embarrassed you are. Your stress or embarrassment levels can also be displayed through an auditory cue generated by one of the other Crickets bus devices such as the MiniMidi Boat or the Voice Recorder. Again we see that with a relatively small set of output/input devices, each of which is carefully integrated into a communicating system, children can build desired wearable or handheld devices easily with a little bit of programming.

\subsection{Going Places}

Equipped with pressure, bend sensor, and shock sensor, children can turn an exercise session into a study of their own body.

In one example, children can design their own pedometers by monitoring the readings from sensors that they embed in their shoes. In addition to recording the sensor values for determining the speed and the total distance traveled during the exercise session, the sensors' values can be used to generate music as they walk or can be studied to learn about the details of one's walking habits. Of course, any of these measured or calculated values can be displayed on the 3-digit display.

In another example, children can use the shoe sensors to measure how much time they are in the air when they jump — the famous "hang time" of pro basketball players. This is easily done with the combination of a touch sensor and near-millisecond precise timing of the Cricket. 


\subsection{Life in the Fast Lane}

In a workshop held at Wellesley College in the summer of 1998, one of the participants designed her own Rollerblade ${ }^{\mathrm{TM}}$ speedometer. She used a magnetic Hall effect sensor affixed to her inline skates, with a magnet wedged in the hub of one of the wheels. Each time the wheel rotated past the Hall effect sensor, a signal was generated. She wrote a program to time the intervals between the signals, transforming it into a velocity measurement. In this sort of project, children gain an appreciation for time/rate/distance thinking that cannot be found in textbook-based school activities.

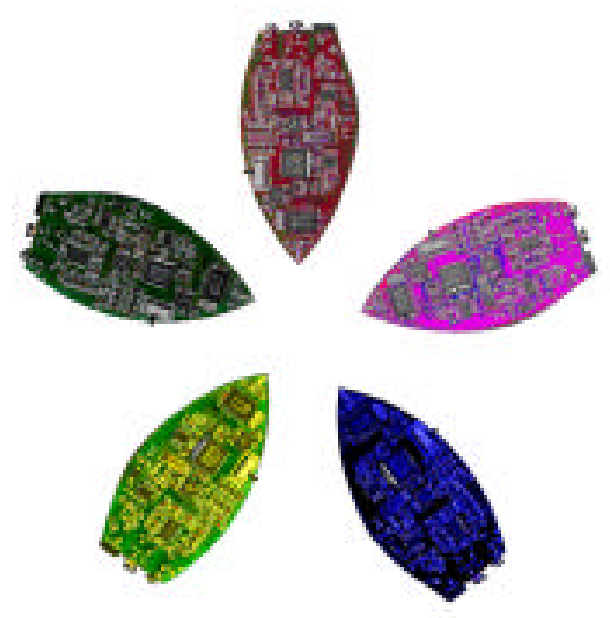

Fig. 6. Musical Friendship Rings

\subsection{Musical Friendship Rings}

A group of five friends get together and build the following functionality into their Cricket-based handheld devices. They all have MIDI boards attached to their Crickets and program their Crickets to send out a unique number to identify itself to the others. Together, they write or download a piece of music with five instruments in it. Each Cricket is programmed to play one of the instruments only. They can play their piece by pressing a button. When they come together, their Crickets handshake and synchronize and play the piece together. In this way they have developed a friendship "ring."

Other variations on this theme could be to also have a round or a fugue and designate one of the Crickets to be the conductor Cricket. If the conductor is present with all the others present then they will play a round. We are currently designing RF bus devices for the Crickets, which would allow for variations of these games. For example, we can expand and vary the interaction radius. This example is just an 
indication of the rich learning and play possibilities in which a group of children collaboratively design the behavior of their handheld/wearable computers.

\subsection{Reach Out and Touch Something}

With the Motorola ${ }^{\circledR}$ CreataLink ${ }^{\mathrm{TM}}$ interface, Crickets can send and receive short data messages over a wireless pager network. This means that a Cricket can originate a page or e-mail, or it can receive instructions sent by page or email. This opens up many possibilities for remote monitoring and control of on-going experiments or other activities. For instance, a child could set up apparatus in the woods behind their school for measuring and reporting on daily temperature, light levels, and other environmental data.

\subsection{Talking to Things, Big and Small}

The last application points to a much richer range of possibilities for the Crickets. With enhanced and multiple communication capabilities, Crickets will be able to talk to other handheld devices, big and small:

- games like Gameboy $^{\mathrm{TM}}$ and Tamagochi ${ }^{\mathrm{TM}}$,

- communication tools like Friend.link ${ }^{\mathrm{TM}}$, Groove Connection ${ }^{\mathrm{TM}}$, and cellular phones

- programmable calculators

- personal musical devices like Walkman ${ }^{\mathrm{TM}}$, Discman ${ }^{\mathrm{TM}}$, or Watchman ${ }^{\mathrm{TM}}$

- home appliances and entertainment units

- and all other Cricket-based or tagged devices in the environment

We think of Cricket-like computational objects as an addition to the existing and growing collection of handheld and wearable devices that not only expand the range of possibilities, but also more importantly empower our children to open up the systems that surround them and take intellectual ownership of them. We see again and again that children relate to an open collection of devices that can be integrated into a functional unit of their choice in fundamentally different ways. Such construction kits allows them to move beyond being only consumers and become designers of the tools they use.

\section{Evaluation and Future Directions}

\subsection{Evaluation}

Over the last three years, the Cricket technology, Cricket Logo, Logo Blocks, and project ideas have been studied in a variety of settings: 
- workshops for children in which they designed

- robots that, for example, are attracted to walls, avoid edges, seek high temperature areas, or seek light sources

- kinetic sculptures as artistic installations or interactive displays

- visual or auditory displays for the data they accumulate with their own scientific instruments

- activities for the body monitoring sensors

- games similar to the ones that are popular in the kids' culture

- hands-on workshops for mentors and educators from

- a number public schools in Massachusetts, Maine, Rhode Island, Colorado, Minnesota, and Montreal Canada

- the five computer clubhouses ${ }^{2}$, four in United States and one in Germany

- a number of children's and science museums

In these workshops, participants built with the materials, discussed and reflected upon their constructions, and brainstormed about project ideas and application areas for their students.

- classrooms and playgrounds of number of a schools in Cambridge and Boston

We learn valuable lessons from our interactions with children and educators that inform the next designs of the various elements in our construction kit. For example, by working closely with children, we have found that what often prevents many of the younger children from finishing their projects on their own is the syntactical complexity of Cricket Logo for beginners. The Logo Blocks programming environment was designed to provide a gentler entry point.

\subsection{Ongoing Research and Future Directions}

Our ongoing work includes both technology development and learning research. We are planning work with younger children, focusing on materials that will allow 5through 8-year-old children to explore ideas of systems, functions, and mechanism (Resnick, et. al, 1999).

We are also focusing on the following technologies to expand and improve the handheld/wearable construction kit:

- Communication Capabilities_-RF transmitter and receiver bus devices

${ }^{2}$ The first Computer Clubhouse was established in Boston as a part of the Computer Museum a little over five years ago. Clubhouses are after-school centers in which children from underserved communities come together and develop proficiency with a number of computational design and production tools through creating projects that are meaningful to them. 
- More Sensors - to detect identification, position, orientation, and motion characteristics of people and things.

- New Input Devices - A vision system for detecting and tracking objects.

- New Output Devices - LCD displays and small printers similar to that Nintendo introduced for GameBoys ${ }^{\mathrm{TM}}$.

- New programming environments-Enabling children to program their devices with handheld WinCE machines and Nintendo GameBoys ${ }^{\mathrm{TM}}$.

- New programming paradigms-A graphical programming environment in which children will specify the components that they are using and make live connections between their iconic representations to specify the flow of control. The details of the design and implementation of this programming environment will be presented elsewhere.

\section{Conclusion}

One may argue that the approach presented in this paper exasperates the complexity that the proliferation of handheld devices has already introduced into our lives. One might wonder if we should not make special machines that integrate the functional units that we have found to be educationally and socially most appropriate for our children. The answer is not obvious and requires much more discussion.

"For the moment some of us old fogeys may somehow have acquired the special knowledge that makes one a master of the computer, but children know that it is a matter of time before they inherit the machines. They are the computer generation.

What lies behind the love affair? Where is it going?

Can it be guided by the older generation into forms constructive or destructive? Or is its evolution already out of our hands?"

In The Children's Machine, Papert focuses on one aspect of these questions: How does the relationship between children and computers affect learning. We agree with him in that "understanding this relationship will be crucial to our ability to shape the future." We also add that as we move to an era where computers are being integrated into the daily lives our children, we should revisit these questions and broaden frameworks to take advantage of the inevitable enormous ubiquity of these machines. These are very hard problems indeed.

However, in regards to who should be designing the machines for our children and what should these machines be able to do, it is important to keep in mind that the current design of most handheld devices reflect the needs and abilities of (professional) adults. In designing handheld/wearable devices for children, we are not faced with the same constraints. In fact, a genuine recognition of the children's "love affair," styles of play, and their sense of ownership of computers compels us to give children as much control as possible over the configuration and the functionality of their machines. 
Our responsibility is to provide our children with tools to not only probe and critique the existing machines, but also express their expectations and aspirations through their designs. Our sample designs and ideas can guide and influence these designs. Having concrete designs to share and discuss with others provides an excellent opportunity to share and reflect on the current and past design principles. A culture that actively engages in design and discussion of its artifacts is far more likely to not only make better choices about the their uses, but also recognize the true potential of the underlying technology. This is a culture in which we hope to live, play, grow, and learn with our children.

\section{Acknowledgements}

The authors would like to express our appreciation to all of the teachers who have participated in this work, including most especially Martha Greenawalt, Mike Petrich, Karen Wilkinson, Rami Alwan, and Phil Firsenbaum. We would like to recognize the contributions of other members of our research group, including Claudia Urrea, Vanessa Stevens Colella, and Genee Lyn Colobong.

Finally we would like to thank all of the children who have participated in this work. We hope that they got as much out of the experience as did we.

\section{References}

1. Hayward, M. "Lego My Penguin!" in The Elementary Mathematician, vol. 9, no. 4. (1995)

2. Martin, F. Children, Cybernetics, and Programmable Turtles, Master of Science dissertation, MIT Department of Mechanical Engineering. (1988)

3. Martin, F. "Kids Learning Engineering Science Using LEGO and the Programmable Brick," unpublished; presented at the American Educational Research Association. (1996)

4. Martin, F., Mikhak, B., Resnick, M., Silverman, B., Berg, R. "To Mindstorms and Beyond: Evolution of a Construction Kit for Magical Machines," in Robots for Kids edited by Alison Druin and James Hendler, Morgan Kaufmann Publishers, Inc. (In press, 1999)

5. Martin, F. Circuits to Control: Learning Engineering by Designing LEGO Robots. Unpublished PhD dissertation, Massachusetts Institute of Technology. (1994)

6. Papert, S., The Children's Machine: Rethinking School in the Age of the Computer. Basic Books. (1993)

7. Resnick, M., Berg, R., Eisenberg. "Beyond Black Boxes: Bringing Transparency and Aesthetics Back to Scientific Investigations," International Journal of the Learning Sciences. (In press, 1999)

8. Resnick, M., Berg, R., Eisenberg, M., and Martin, F. Learning with Digital Manipulatives: A New Generation of Froebel Gifts for Exploring "Advanced" Mathematical and Scientific Concepts. Proposal to the National Science Foundation. (1999) 


\section{Appendix: Supplementary References}

In this section we provide references to material available on the web about the Cricket technology, the programming environments, and the workshops mentioned in this paper.

Crickets: http://www.media.mit.edu/ fredm/projects/crickets/

Cricket Logo: http://el.www.media.mit.edu/people/mikhak/sd98/CricketLogo.html

Logo Blocks: http://www.media.mit.edu/ fredm/projects/cricket/logo-blocks/

Beyond Black Boxes: http://el.www.media.mit.edu/projects/bbb/

Sensory Design 98: http://el.www.media.mit.edu/people/mikhak/sd98/ 